\title{
Indicadores de rendimiento en los Grand Slams de tenis
}

\author{
Performance Indicators in Tennis Grand Slams
}

\author{
Adrián Escudero-Tena' ${ }^{(1)}$ \\ Abel Castrejon \\ Sergio J. Ibáñez * (i) \\ 1. Grupo de Optimización del Entrenamiento y del Rendimiento Deportivo. Facultad de Ciencias del Deporte. Universidad de Extremadura, España.
}

\begin{abstract}
Resumen
El objetivo de la presente investigación trata de caracterizar las diferencias que se dan en indicadores de rendimiento de los Grand Slams de tenis, con el fin de identificar aquellas conductas técnicas y tácticas que resulten más eficaces en situaciones reales de juego. Se trata de un estudio descriptivo y observacional, de metodología cuantitativa. La muestra estuvo compuesta por 338 partidos, de la categoría individual absoluta masculina en la temporada 2018 de tres torneos Grand Slam (Roland Garros, Wimbledon y Open EEUU). Para ello, se analizaron las diferencias de en los indicadores de rendimiento en diversas variables situacionales (tipo de torneo, resultado del partido, match status y diferencia entre sets) a través de análisis descriptivos e inferenciales. Los resultados mostraron que existen indicadores de rendimiento y diferencias significativas en los torneos analizados en cuanto al tipo de torneo, resultado del partido, match status y en menor medida en la diferencia entre sets. Concluyendo que los indicadores de rendimiento son propios de cada torneo o superficie de juego, que la importancia de llevar la iniciativa es fundamental para ganar en tenis y que la forma de juego no varía según va transcurriendo el partido. Estos hallazgos resultan de gran interés, pues condicionan su puesta a punto para cada torneo y el juego de los tenistas, estableciéndose diferentes estrategias de competición.
\end{abstract}

Palabras clave: Indicadores de rendimiento, competición, superficie de juego, resultado, tenis.

\begin{abstract}
The objective of this research is to characterize the differences in performance indicators of Grand Slam tennis, in order to identify those technical and tactical behaviors that are more effective in real life playing situations. It is a descriptive and observational study, with a quantitative methodology. The sample was composed of 338 matches, of the absolute individual male category in the 2018 season of three Grand Slam tournaments (Roland Garros, Wimbledon and US Open). The differences in performance indicators in various situational variables (type of tournament, match result, match status and difference between sets) were analyzed through descriptive and inferential analysis. The results showed that there are significant performance indicators and differences in the analyzed tournaments in terms of tournament type, match result, match status and to a lesser extent in the difference between sets. We concluded that performance indicators are specific to each tournament or playing surface, that the importance of taking the initiative is fundamental to winning in tennis and that the form of play does not vary as the match progresses. These findings are of great interest, as they condition their setting for each tournament and the play of the players, establishing different competition strategies.
\end{abstract}

Keywords: Performance indicators, competition, playing surface, score, tennis.

*Autor de correspondencia: Sergio J. Ibáñez, sibanez@unex.es

Recibido: 11 de mayo de 2020

Aceptado: 1 de junio de 2020

Publicado: 14 de julio de 2020

Como citar (APA): Escudero-Tena, A., Castrejon, A., \& Ibáñez, S. J. (2020). Indicadores de rendimiento en los Grand Slams de tenis. JUMP, (2), 26-36. https://doi.org/10.17561/jump.n2.3

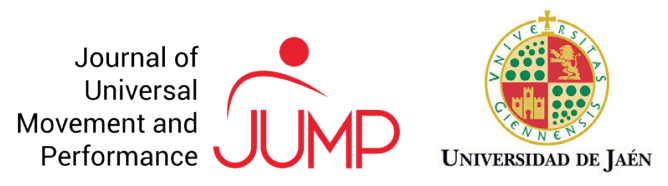




\section{Introducción}

El tenis es considerado uno de los principales deportes de raqueta (Lees, 2003; Akpinar, Devrilmez, \& Kirazci, 2012), además de ser objeto de estudio de diversos investigadores (Ferrauti, Bergeron, Pluim, \& Weber, 2001; O’Donoghue \& Ingram, 2001; Smekal, et al., 2001). Uno de los aspectos más investigados en este deporte es la descripción de la competición y el descubrimiento de indicadores de rendimiento a través del análisis de juego con el fin de recopilar conocimientos válidos, precisos y fiables (Hughes \& Franks, 2007). Más concretamente, se centran en descubrir indicadores de rendimiento relacionados con el tipo de juego (Martínez-Gallego, et al., 2013; O'Donoghue \& Ingram, 2001), condición física (Fernández-Fernández, Kinner \& Ferrauti, 2010), aspectos técnicos (Brown \& O'Donoghue, 2008), tácticos (Gillet, Leroy, Thouvarecq \& Stein, 2009; Reid, McMurtrie \& Crespo, 2010; Piles \& Crespo, 2012) y psicológicos (Cowden, Fuller \& Anshel, 2014; Kovacs, 2007).

Los indicadores de rendimiento pueden variar por diversos factores, en tenis uno de esos factores es el tipo de torneo y superficie (hierba, pista dura o tierra batida) de las pistas donde se juegan los partidos (Cross \& Pollard, 2009; FernándezGarcía, Torres-Luque, Sánchez-Pay \& Fradua, 2012; Filipcic, Filipcic \& Berendijas, 2008; Barnett \& Pollard, 2007). El porcentaje de la eficacia de conseguir punto es superior cuando se entra el primer servicio en superficie de hierba (Filipcic et al., 2008; Fernández-García et al., 2012). Además en superficie de hierba la velocidad del primer y del segundo servicio es superior, el número de aces es mayor y las subidas a la red se dan en más ocasiones en hierba que en los demás tipos de superficie (Barnett \& Pollard, 2007; FernándezGarcía et al., 2012). En cambio, la eficacia de conseguir puntos al resto, las oportunidades de rotura del servicio y los errores cometidos son superiores en tierra batida (Barnett \& Pollard, 2007; Fernández-García et al., 2012). O'Donoghue e Ingram (2001) identificaron que en el número de dobles faltas era superior en pistas de hierba, mientras que Fernández-García et al. (2012) no encontraron diferencias significativas entre las diferentes superficies de juego.

Diversos estudios concluyen en sus investigaciones que el estilo de juego de los jugadores cambia en función del tipo de superficie.
Martínez-Gallego et al. (2013), O’Donoghue e Ingram (2001), Furlong (1995), Barnett y Pollard (2007) y Fernández-García et al. (2012) afirman en sus investigaciones que el juego es más rápido en césped y en pista dura, los puntos tienen un menor número de golpes, los partidos tienen una duración menor y predomina el estilo de juego ofensivo o cerca de la red. Por el contrario, O'Donoghue e Ingram, (2001), Unierzyski y Wieczorek (2004), Fernández, Méndez, Pluim, Fernández-García y Terrados (2006), Barnett y Pollard (2007) y Fernández-García et al. (2012), concluyen en sus estudios que el juego es más lento en tierra batida, el rally es mayor, los partidos tienen una duración mayor y predomina el estilo de juego defensivo o desde el fondo de la pista.

Por otro lado, existe una diferencia significativa de indicadores de rendimiento entre los tenistas que ganan y los que pierden un partido. Filipcic et al. (2008) estudian la diferencia entre indicadores de rendimiento durante el Roland Garros de 2005 según el género y resultado del partido, concluyendo que los jugadores masculinos que ganan los partidos obtienen un número superior de aces, puntos ganados en posiciones cercanas a la red, puntos ganados con primer y segundo servicio, porcentaje de primeros servicios, total de puntos ganados y número de winners, mientras que los jugadores que pierden los partidos realizan más dobles faltas y errores no forzados. Sin embargo, en categoría femenina, las jugadoras que ganan los partidos obtienen un número superior de puntos de break convertidos, puntos ganados en posiciones cercanas a la red, puntos ganados con primer y segundo servicio, porcentaje de primeros servicios, total de puntos ganados y número de winners, mientras que las jugadoras que pierden los partidos realizan más dobles faltas y errores no forzados (Filipcic et al., 2008). Katić, Milat, Zagorac \& Đurović (2011) indican que los tenistas masculinos ganadores tienen un mayor número de aces, puntos logrados con el primer y segundo servicio y puntos ganados durante el servicio del oponente en primeras rondas. Sin embargo, los jugadores que pierden los partidos muestran una tasa mayor de dobles faltas y errores no forzados (Katić et al., 2011). Por su parte, Martínez-Gallego et al. (2013) determinan en su trabajo que los jugadores profesionales que ganan los partidos pasan menos tiempo en las zonas defensivas en comparación a los jugadores que los pierden. De igual forma, los ganadores 
recorren más distancia durante el tiempo activo de juego que los perdedores (Martínez-Gallego et al., 2013). Sánchez-Pay, Torres-Luque, FernándezGarcía, Sanz-Rivas \& Andrés (2017) analizaron los partidos masculinos individuales de los Juegos Paralímpicos de 2012, donde los jugadores ganadores tuvieron valores significativamente más altos en aces, porcentaje de primer servicio y puntos ganados en primer y segundo servicio, mientras que los jugadores que perdieron los partidos obtuvieron valores superiores en dobles faltas y puntos jugados con el segundo servicio.

Son escasos los estudios sobre tenis que hagan referencia hacia como el match status o estado del marcador interviene en el partido. Cui, Liu, Liu \& Gómez (2019) analizaron 145 partidos individuales de Grand Slam en el cuadro principal masculino, demostrando que los jugadores con un resultado puntual a favor, negativo o empate ganan más puntos de los que pierden, siendo dicha afirmación únicamente significativa cuando el resultado puntual es a favor. Además, los jugadores ganan un mayor número de puntos cuando el resultado puntual en su servicio es negativo, mientras que pierden un mayor número de puntos cuando el resultado puntual durante el resto es positivo.

Se han encontrado investigaciones de otros deportes como el fútbol (Jones, James \& Mellalieu, 2004; Bloomfield, Polman \& O'Donoghue, 2005; Lago \& Martín, 2007), el baloncesto (Courel, Robert, Ortega \& Cárdenas, 2014; Gómez, Alarcón-López, \& Ortega-Toro, 2015) o el voleibol (Afonso, Mesquita \& Palao, 2005; Monteiro, Mesquita \& Marcelino, 2009) donde el match status ha influido en el análisis del juego. En futbol, se han identificado relaciones entre el porcentaje de posesión del balón y el estado del marcador, siendo mayor la posesión del balón cuando el equipo va perdiendo (Jones, James \& Mellalieu, 2004). Por su parte, Lago y Martín (2007) concluyeron que los equipos locales muestran mayor posesión del balón cuando el resultado parcial es empate. Además, Bloomfield et al., (2005) afirman en su estudio que los estilos de juego cambian en función del marcador. En baloncesto, los equipos que van delante en el marcador realizan menos bloqueos indirectos, arriesgan menos en los pases y sus posesiones son más largas (Courel et al., 2014; Gómez et al., 2015). Por último, en voleibol un resultado parcial positivo del partido interviene de forma directa en una mayor eficacia de la zona defensiva lo que produce a su vez una posterior influencia en la colocación y el ataque (Afonso et al., 2005).

El análisis del rendimiento deportivo se aplia para recopilar información precisa, la cual permite extraer datos de comportamientos espontáneos y en situaciones reales de juego. Dichos datos proporcionan información objetiva que resulta de gran interés para el diseño de tareas específicas de entrenamiento, el desarrollo de estrategias de competición, la aplicación de feedback o la mejora de la toma de decisiones del deportista. Tras el análisis de la literatura se constata la escasez de estudios en tenis sobre las diferencias de indicadores de rendimiento teniendo en cuenta una serie de variables situacionales como el tipo de torneo y su superficie, resultado del partido, match status y su diferencia entre sets. Por ello, la presente investigación pretende identificar las diferencias existentes entre los indicadores de rendimiento en tres Grand Slams de tenis (Roland Garros, Wimbledon y Open de EEUU), así como el comportamiento de los tenistas ante las diferentes situaciones de juego. Los objetivos de la investigación fueron: i) identificar las diferencias entre los diferentes torneo en los indicadores de rendimiento; ii) analizar las diferencias en los indicadores de rendimiento en función del resultado del partido; iii) comprobar las diferencias en los indicadores de rendimiento atendiendo al match status; iv) identificar las diferencias en los indicadores de rendimiento en función de la diferencia en el orden de los set.

\section{Método}

Según Montero y León (2007), la metodología de la investigación es cuantitativa y más específicamente es un estudio descriptivo mediante un código arbitrario de observación natural. Además, este trabajo fue empírico, nomotético, transversal y multidimensional (Ato, López \& Benavente, 2013).

Los participantes del estudio fueron los tenistas masculinos que compitieron en categoría absoluta en los Grand Slams de tenis durante la temporada 2018. Se analizaron los partidos de 3 torneos, concretamente 338 partidos de los torneos Roland Garros $(n=126)$, Wimbledon $(n=127)$ y Open de Estados Unidos $(n=85)$.

Según Lago (2009) las variables registradas en este estudio fueron variables situacionales, 
variables relacionadas con el contexto del juego, las cuales pueden influir y condicionar el juego de un jugador o de un equipo. Así, las variables independientes fueron tipo de torneo (Roland Garros, Wimbledon u Open EEUU), resultado del partido (ganado o perdido), match status o resultado parcial del partido cada juego (ganando, perdiendo o empatando) y diferencia entre sets (primero, segundo, tercero, cuarto y quinto set).

Las variables dependientes o indicadores de rendimiento fueron: el número de aces, número de dobles faltas, porcentaje de primeros servicios, puntos ganados con el primer servicio, puntos ganados con el segundo servicio, puntos de break salvados, puntos ganados en la primera devolución, puntos ganados en la segunda devolución, puntos de break ganados, número de golpes ganadores, errores no forzados, puntos ganados con el servicio, puntos ganados con el resto, total de puntos ganados, juegos ganados con el servicio, juegos ganados con el resto y total de juegos ganados.

El instrumento empleado en la investigación fue una hoja de observación "ad hoc", a medida para el presente estudio como recomiendan Anguera y Hernández-Mendo (2016), la cual se empleó para la recogida de datos.
Tras una revisión bibliográfica, se ideó el planteamiento del problema y se construyó una hoja de observación "ad hoc". Una vez definidas las variables y categorías, los datos fueron obtenidos de la página oficial https://www.mismarcadores.com, de las páginas oficiales de los tres torneos en cuestión https://www.rolandgarros.com/en-us/, https:// www.wimbledon.com/index.html y https://www. usopen.org/index.html y de la página oficial de la ATP (Asociación de Tenistas Profesionales) https:// www.atptour.com/. Así, tras la identificación de cada variable se registró en una hoja Excel el número de veces que se dieron o la categoría observada en cada juego de los partidos analizados.

Los análisis de datos facilitaron la toma de decisiones durante del proceso de investigación, ayudando a la resolución de los objetivos. En primer lugar, se realizaron pruebas de asunción de criterios para determinar el modelo de contraste de hipótesis a emplear. Se comprobó que ninguno de los indicadores de rendimiento registrados cumple con los criterios de normalidad (Cubo, 2011). Por ello, el modelo de contraste de hipótesis fue no paramétrico (Tabla 1).

Se realizó un análisis descriptivo para obtener la frecuencia y porcentaje de veces que se produjeron las variables o sus categorías. Se empleó la media

Tabla 1. Resultados de la prueba de asunción de criterios (Kolmogorov-Smimov)

\begin{tabular}{|c|c|c|c|c|c|c|c|}
\hline \multirow[t]{2}{*}{ Variables dependientes } & \multicolumn{2}{|c|}{$\mathrm{RG}$} & \multicolumn{2}{|c|}{ W } & \multicolumn{2}{|c|}{ EEUU } & \multirow[t]{2}{*}{ Toma decisión } \\
\hline & K-S & $\mathrm{p}$ & $\mathrm{K}-\mathrm{S}$ & $\mathrm{p}$ & $\mathrm{K}-\mathrm{S}$ & $\mathrm{p}$ & \\
\hline Aces & .192 & .000 & .174 & .000 & .172 & .000 & NP \\
\hline Dobles faltas & .227 & .000 & .235 & .000 & .230 & .000 & NP \\
\hline$\%$ primer servicio & .057 & .000 & .037 & .004 & .049 & .001 & NP \\
\hline Puntos ganados 1er servicio & .063 & .000 & .056 & .000 & .052 & .001 & NP \\
\hline Puntos ganados 2 do servicio & .053 & .000 & .054 & .000 & .061 & .000 & NP \\
\hline Puntos de break salvados & .214 & .000 & .263 & .000 & .249 & .000 & NP \\
\hline Puntos ganados $1^{\text {a }}$ devolución & .061 & .000 & .058 & .000 & .054 & .000 & NP \\
\hline Puntos ganados $2^{a}$ devolución & .064 & .000 & .245 & .000 & .201 & .000 & NP \\
\hline Puntos de break ganados & .205 & .000 & .245 & .000 & .201 & .000 & NP \\
\hline Golpes ganadores & .090 & .000 & .096 & .000 & .201 & .000 & NP \\
\hline Errores no forzados & .087 & .000 & .095 & .000 & .089 & .000 & NP \\
\hline Puntos ganados con el servicio & .046 & .000 & .053 & .000 & .051 & .000 & NP \\
\hline Puntos ganados con el resto & .048 & .000 & .050 & .000 & .050 & .001 & NP \\
\hline Total de puntos ganados & .049 & .000 & .035 & .008 & .054 & .000 & NP \\
\hline Juegos ganados con el servicio & .195 & .000 & .238 & .000 & .186 & .000 & NP \\
\hline Juegos ganados con el resto & .194 & .000 & .239 & .000 & .186 & .000 & NP \\
\hline Total de juegos ganados & .084 & .000 & .113 & .000 & .084 & .000 & NP \\
\hline
\end{tabular}

$p<.05$ 
y la desviación típica con el objetivo de describir el comportamiento de las variables dependientes según los valores de las variables independientes.

Se prosiguió con un análisis inferencial para analizar las asociaciones entre las variables, mediante tablas de contingencia, incluyendo la prueba $U$ de Mann-Whitney y la prueba $\mathrm{H}$ de Kruskal-Wallis.

Los análisis estadísticos se realizaron con el software SPSS v.21 (IBM Corp. Lanzado en 2012. IBM SPSS Statistics para Windows, NY: IBM Corp). La significación estadística se estableció en $p<$ 0,05 .

\section{Resultados}

La tabla 2 recoge los resultados descriptivos y las diferencias en función del tipo de torneo en los indicadores de rendimiento.

Los resultados muestran diferencias significativas en todos los indicadores de rendimiento entre los tres torneos analizados excepto en los puntos de break salvados, total de puntos ganados y total de juegos ganados.

En Wimbledon la media de las variables aces, porcentaje de primer servicio, puntos ganados en 1er y 2nd servicio, golpes ganadores y puntos y juegos ganados con el servicio es superior que la de los otros dos torneos. Por otro lado, en Roland Garros, la media de puntos de break salvados, puntos ganados con $1^{\mathrm{a}}$ y $2^{\mathrm{a}}$ devolución, errores no forzados y puntos y juegos ganados al resto es superior. Por último, la media de dobles faltas y puntos de break ganados es superior en el Open EEUU.

La tabla 3 presenta los resultados descriptivos y las diferencias en función del resultado del partido en los indicadores de rendimiento en cada uno de los torneos analizados.

Los resultados muestran diferencias significativas entre todas las variables dependientes en los tres torneos analizados con respecto al resultado del partido, excepto en los puntos de break salvados.

Tabla 2. Resultados descriptivos y diferencias del tipo de torneo en los indicadores de rendimiento

\begin{tabular}{|c|c|c|c|c|c|c|c|c|c|}
\hline \multirow[t]{2}{*}{ Variables dependientes } & \multicolumn{2}{|c|}{$R G$} & \multicolumn{2}{|c|}{ W } & \multicolumn{2}{|c|}{ EEUU } & \multicolumn{3}{|c|}{ Total } \\
\hline & $\bar{x}$ & DT & $\bar{X}$ & DT & $\bar{x}$ & DT & $H$ & $g l$ & $p$ \\
\hline Aces & 1.79 & 1.87 & 3.24 & 2.99 & 2.63 & 2.22 & 160.03 & 2 & .000 \\
\hline Dobles faltas & 1.05 & 1.12 & 1.23 & 1.15 & 1.36 & 1.30 & 25.758 & 2 & .000 \\
\hline$\%$ primer servicio & 0.60 & 0.11 & 0.63 & 0.10 & 0.60 & 0.11 & 30.160 & 2 & .000 \\
\hline Puntos ganados 1er servicio & 0.70 & 0.14 & 0.75 & 0.13 & 0.72 & 0.14 & 45.047 & 2 & .000 \\
\hline $\begin{array}{l}\text { Puntos ganados } 2 \mathrm{do} \\
\text { servicio }\end{array}$ & 0.50 & 0.17 & 0.53 & 0.18 & 0.51 & 0.17 & 6.641 & 2 & .036 \\
\hline Puntos de break salvados & 0.44 & 0.36 & 0.43 & 0.39 & 0.42 & 0.37 & 2.083 & 2 & .353 \\
\hline $\begin{array}{l}\text { Puntos ganados } 1^{\mathrm{a}} \text { devo- } \\
\text { lución }\end{array}$ & 0.30 & 0.14 & 0.25 & 0.13 & 0.28 & 0.14 & 43.311 & 2 & .000 \\
\hline $\begin{array}{l}\text { Puntos ganados } 2^{a} \text { devo- } \\
\text { lución }\end{array}$ & 0.40 & 0.17 & 0.36 & 0.17 & 0.37 & 0.16 & 20.787 & 2 & .000 \\
\hline Puntos de break ganados & 0.36 & 0.34 & 0.32 & 0.36 & 0.37 & 0.35 & 14.097 & 2 & .001 \\
\hline Golpes ganadores & 9.57 & 4.50 & 10.5 & 5.41 & 10.1 & 4.86 & 14.089 & 2 & .001 \\
\hline Errores no forzados & 10.7 & 5.18 & 7.61 & 4.19 & 10.3 & 4.99 & 217.49 & 2 & .000 \\
\hline $\begin{array}{l}\text { Puntos ganados con el } \\
\text { servicio }\end{array}$ & 0.62 & 0.12 & 0.66 & 0.12 & 0.63 & 0.12 & 48.657 & 2 & .000 \\
\hline Puntos ganados con el resto & 0.37 & 0.12 & 0.33 & 0.12 & 0.36 & 0.12 & 48.621 & 2 & .000 \\
\hline Total de puntos ganados & 0.50 & 0.10 & 0.50 & 0.08 & 0.50 & 0.09 & 0.001 & 2 & 1.00 \\
\hline $\begin{array}{l}\text { Juegos ganados con el } \\
\text { servicio }\end{array}$ & 0.75 & 0.25 & 0.81 & 0.21 & 0.76 & 0.23 & 32.952 & 2 & .000 \\
\hline $\begin{array}{l}\text { Juegos ganados con el } \\
\text { resto }\end{array}$ & 0.25 & 0.25 & 0.19 & 0.04 & 0.23 & 0.23 & 33.404 & 2 & .000 \\
\hline Total de juegos ganados & 0.50 & 0.20 & 0.50 & 0.17 & 0.49 & 0.03 & 0.005 & 2 & .997 \\
\hline
\end{tabular}


Tabla 3. Resultados descriptivos y las diferencias en función del resultado del partido en cada torneo

\begin{tabular}{|c|c|c|c|c|c|c|c|c|c|c|c|c|}
\hline \multirow{3}{*}{$\begin{array}{c}\text { Variables } \\
\text { dependientes }\end{array}$} & \multicolumn{4}{|c|}{ Roland Garros } & \multicolumn{4}{|c|}{ Wimbledon } & \multicolumn{4}{|c|}{ Open EEUU } \\
\hline & \multirow{2}{*}{$\frac{\mathrm{G}}{\bar{X}}$} & \multirow{2}{*}{$\begin{array}{l}\mathrm{P} \\
\bar{x}\end{array}$} & \multicolumn{2}{|c|}{ Total } & \multirow{2}{*}{$\frac{\mathrm{G}}{\bar{X}}$} & \multirow{2}{*}{$\frac{\mathrm{P}}{\bar{X}}$} & \multicolumn{2}{|c|}{ Total } & \multirow{2}{*}{$\frac{\mathrm{G}}{\bar{X}}$} & \multirow{2}{*}{$\frac{P}{\bar{X}}$} & \multicolumn{2}{|c|}{ Total } \\
\hline & & & $U$ & $p$ & & & $U$ & $p$ & & & $U$ & $p$ \\
\hline Aces & 2.0 & 1.5 & 91.181 .5 & .000 & 3.9 & 2.6 & 39.909 .0 & .001 & 3.0 & 2.3 & 39.909 .0 & .001 \\
\hline Dobles faltas & 0.9 & 1.2 & 123.058 .5 & .003 & 1.1 & 1.4 & 54.126 .0 & .001 & 1.3 & 1.5 & 54.126 .0 & .001 \\
\hline \%Primer servicio & 0.6 & 0.6 & 100.732 .0 & .011 & 0.6 & 0.6 & 41.003 .0 & .005 & 0.6 & 0.6 & 41.003 .0 & .005 \\
\hline $\begin{array}{l}\text { Puntos ganados } \\
\text { ler servicio }\end{array}$ & 0.8 & 0.6 & 56.623 .0 & .000 & 0.8 & 0.7 & 29.088 .5 & .000 & 0.8 & 0.7 & 29.088 .5 & .000 \\
\hline $\begin{array}{l}\text { Puntos ganados } \\
\text { 2nd servicio }\end{array}$ & 0.6 & 0.4 & 69.972 .5 & .000 & 0.6 & 0.5 & 31.177 .5 & .000 & 0.6 & 0.5 & 31.177 .5 & .000 \\
\hline $\begin{array}{l}\text { Puntos de break } \\
\text { salvados }\end{array}$ & 0.4 & 0.5 & 114.648 .0 & .427 & 0.4 & 0.5 & 48.516 .5 & .513 & 0.4 & 0.4 & 48.516 .5 & .513 \\
\hline $\begin{array}{l}\text { Puntos ganados } \\
1^{\text {a }} \text { devolución }\end{array}$ & 0.4 & 0.2 & $57,202.5$ & .000 & 0.3 & 0.2 & 28.993 .5 & .000 & 0.3 & 0.2 & 28.993 .5 & .000 \\
\hline $\begin{array}{l}\text { Puntos ganados } \\
2^{\text {a }} \text { devolución }\end{array}$ & 0.5 & 0.4 & 74.321 .0 & .000 & 0.4 & 0.3 & 33.361 .5 & .000 & 0.4 & 0.3 & 33.361 .5 & .000 \\
\hline $\begin{array}{l}\text { Puntos de break } \\
\text { ganadores }\end{array}$ & 0.5 & 0.2 & 63.070 .0 & .000 & 0.4 & 0.2 & 27.506 .5 & .000 & 0.5 & 0.3 & 27.506 .5 & .000 \\
\hline Golpes ganadores & 10.4 & 8.7 & 84.296 .5 & .000 & 11.6 & 9.5 & 37.936 .0 & .000 & 10.9 & 9.4 & 37.936 .0 & .000 \\
\hline $\begin{array}{l}\text { Errores no forza- } \\
\text { dos }\end{array}$ & 9.6 & 11.8 & 141.437 .5 & .000 & 6.7 & 8.5 & 56.782 .5 & .000 & 9.4 & 11.2 & 56.782 .5 & .000 \\
\hline $\begin{array}{l}\text { Puntos ganados } \\
\text { con el servicio }\end{array}$ & 0.7 & 0.6 & 47.044 .0 & .000 & 0.7 & 0.6 & 23.747 .0 & .000 & 0.7 & 0.6 & 23.747 .0 & .000 \\
\hline $\begin{array}{l}\text { Puntos ganados } \\
\text { con el resto }\end{array}$ & 0.4 & 0.3 & 47.124 .5 & .000 & 0.4 & 0.3 & 23.794 .0 & .000 & 0.4 & 0.3 & 23.794 .0 & .000 \\
\hline $\begin{array}{l}\text { Total de puntos } \\
\text { ganados }\end{array}$ & 0.6 & 0.4 & 33.809 .5 & .000 & 0.6 & 0.5 & 15.592 .5 & .000 & 0.6 & 0.4 & 15.592 .5 & .000 \\
\hline $\begin{array}{l}\text { Juegos ganados } \\
\text { con el servicio }\end{array}$ & 0.9 & 0.6 & 52.414 .0 & .000 & 0.9 & 0.7 & 22.199 .0 & .000 & 0.9 & 0.7 & 22.199 .0 & .000 \\
\hline $\begin{array}{l}\text { Juegos ganados } \\
\text { con el resto }\end{array}$ & 0.4 & 0.1 & 52.459 .0 & .000 & 0.3 & 0.1 & 22.101 .0 & .000 & 0.3 & 0.1 & 22.101 .0 & .000 \\
\hline $\begin{array}{l}\text { Total de juegos } \\
\text { ganados }\end{array}$ & 0.6 & 0.4 & 39.149 .5 & .000 & 0.6 & 0.4 & 15.625 .0 & .000 & 0.6 & 0.4 & 15.625 .0 & .000 \\
\hline
\end{tabular}

$p<.05 ; G=$ ganado; $P=$ perdido

Los tenistas que ganan los partidos obtienen de manera general una media superior en los tres torneos en todas las variables dependientes frente a los jugadores que pierden, excepto en las dobles faltas, puntos de break salvados y en los errores no forzados.

Los jugadores que ganan obtienen en Wimbledon la media de las variables aces y golpes ganadores superior a la de los otros dos torneos. Por otro lado, en Roland Garros, la media de los puntos ganados con $1^{\text {a }}$ y $2^{\text {a }}$ devolución, los errores no forzados y los juegos ganados al resto es superior en los tenistas que ganan. Por último, la media de los tenistas que ganan los partidos obtienen una media superior en las dobles faltas en el Open EEUU.
La tabla 4 presenta los resultados descriptivos y las diferencias en función del match status duranteel partido en los indicadores de rendimiento en cada torneo analizado.

Se identifican diferencias significativas entre todas las variables dependientes en los tres torneos analizados con respecto al match status, excepto en las dobles faltas, el porcentaje de primer servicio y los puntos de break salvados.

Los jugadores que van ganando los partidos obtienen de manera general una media superior en los tres torneos en todas las variables dependientes seguido de los que van empatando y por último los que van perdiendo, excepto en las dobles faltas, puntos de break salvados y en los errores no forzados. 
Tabla 4. Resultados descriptivos y diferencias en función del match status con respecto a los indicadores de rendimiento

\begin{tabular}{|c|c|c|c|c|c|c|c|c|c|c|c|c|c|c|c|}
\hline \multirow[t]{3}{*}{$\begin{array}{c}\text { Variables } \\
\text { dependientes }\end{array}$} & \multicolumn{5}{|c|}{ Roland Garros } & \multicolumn{5}{|c|}{ Wimbledon } & \multicolumn{5}{|c|}{ Open EEUU } \\
\hline & \multirow{2}{*}{$\frac{\mathrm{G}}{\bar{X}}$} & \multirow{2}{*}{$\frac{\mathrm{P}}{\bar{x}}$} & \multirow{2}{*}{$\begin{array}{l}\mathrm{E} \\
\bar{X}\end{array}$} & \multicolumn{2}{|c|}{ Total } & \multirow{2}{*}{$\begin{array}{l}G \\
\bar{X}\end{array}$} & \multirow{2}{*}{$\begin{array}{l}\mathrm{P} \\
\bar{x}\end{array}$} & \multirow{2}{*}{$\frac{\mathrm{E}}{\bar{X}}$} & \multicolumn{2}{|c|}{ Total } & \multirow{2}{*}{$\frac{G}{\bar{X}}$} & \multirow{2}{*}{$\frac{\mathrm{P}}{\bar{X}}$} & \multirow{2}{*}{$\frac{E}{\bar{X}}$} & \multicolumn{2}{|c|}{ Total } \\
\hline & & & & $H$ & $p$ & & & & $H$ & $p$ & & & & $H$ & $p$ \\
\hline Aces & 2.1 & 1.5 & 1.8 & 16.0 & .000 & 3.9 & 2.6 & 3.2 & 32.5 & .000 & 2.9 & 2.2 & 2.8 & 11.7 & .003 \\
\hline Dobles faltas & 1.0 & 1.2 & 1.0 & 4.90 & .086 & 1.2 & 1.3 & 1.2 & 1.57 & .455 & 1.3 & 1.4 & 1.4 & 1.33 & .514 \\
\hline \%Primer servicio & 0.6 & 0.6 & 0.6 & 1.07 & .585 & 0.6 & 0.6 & 0.6 & 4.04 & .132 & 0.6 & 0.6 & 0.6 & 1.13 & .567 \\
\hline $\begin{array}{l}\text { P. ganados 1er } \\
\text { servicio }\end{array}$ & 0.8 & 0.6 & 0.7 & 63.1 & .000 & 0.8 & 0.7 & 0.7 & 64,1 & .000 & 0.8 & 0.7 & 0.7 & 39.8 & .000 \\
\hline $\begin{array}{l}\text { P. ganados 2nd } \\
\text { servicio }\end{array}$ & 0.5 & 0.5 & 0.5 & 31.3 & .000 & 0.6 & 0.5 & 0.5 & 15.2 & .000 & 0.6 & 0.5 & 0.5 & 14.1 & .001 \\
\hline $\begin{array}{l}\text { P. de break sal- } \\
\text { vados }\end{array}$ & 0.4 & 0.5 & 0.4 & 1.03 & .596 & 0.4 & 0.5 & 0.4 & 4.28 & .118 & 0.4 & 0.4 & 0.4 & 3.57 & .168 \\
\hline $\begin{array}{l}\text { P. ganados } 1^{\text {a }} \\
\text { devolución }\end{array}$ & 0.3 & 0.2 & 0.3 & 60.4 & .000 & 0.3 & 0.2 & 0.2 & 64.1 & .000 & 0.3 & 0.2 & 0.3 & 40.0 & .000 \\
\hline $\begin{array}{l}\text { P. ganados } 2^{\text {a }} \\
\text { devolución }\end{array}$ & 0.4 & 0.4 & 0.4 & 22.9 & .000 & 0.4 & 0.3 & 0.4 & 16.2 & .000 & 0.4 & 0.3 & 0.4 & 11.2 & .004 \\
\hline $\begin{array}{l}\text { P. de break ga- } \\
\text { nadores }\end{array}$ & 0.4 & 0.3 & 0.4 & 37.3 & .000 & 0.4 & 0.2 & 0.3 & 27,1 & .000 & 0.4 & 0.3 & 0.4 & 17.5 & .000 \\
\hline Golpes ganadores & 10.5 & 8.8 & 9.5 & 22.5 & .000 & 11.8 & 9.6 & 10.2 & 39.0 & .000 & 10.5 & 9.5 & 10.3 & 6.05 & .048 \\
\hline Errores no forzados & 10.0 & 11.2 & 10.8 & 10.2 & .006 & 7.0 & 8.2 & 7.6 & 10.7 & .005 & 9.3 & 10.7 & 10.7 & 9.48 & .009 \\
\hline $\begin{array}{l}\text { P. ganados con el } \\
\text { servicio }\end{array}$ & 0.7 & 0.6 & 0.6 & 73.5 & .000 & 0.7 & 0.6 & 0.7 & 62.7 & .000 & 0.7 & 0.6 & 0.6 & 47.3 & .000 \\
\hline $\begin{array}{l}\text { P. ganados con el } \\
\text { resto }\end{array}$ & 0.4 & 0.3 & 0.4 & 73.0 & .000 & 0.4 & 0.3 & 0.3 & 64.7 & .000 & 0.4 & 0.3 & 0.4 & 46.8 & .000 \\
\hline $\begin{array}{l}\text { Total de puntos } \\
\text { ganados }\end{array}$ & 0.5 & 0.5 & 0.5 & 92.6 & .000 & 0.5 & 0.5 & 0.5 & 82.1 & .000 & 0.5 & 0.5 & 0.5 & 63.9 & .000 \\
\hline $\begin{array}{l}\text { J. ganados con el } \\
\text { servicio }\end{array}$ & 0.8 & 0.7 & 0.7 & 62.6 & .000 & 0.9 & 0.8 & 0.8 & 41.3 & .000 & 0.9 & 0.7 & 0.8 & 45.5 & .000 \\
\hline $\begin{array}{l}\text { J. ganados con el } \\
\text { resto }\end{array}$ & 0.3 & 0.2 & 0.3 & 65.4 & .000 & 0.2 & 0.1 & 0.2 & 40.5 & .000 & 0.3 & 0.1 & 0.2 & 45.0 & .000 \\
\hline $\begin{array}{l}\text { Total de juegos } \\
\text { ganados }\end{array}$ & 0.6 & 0.4 & 0.5 & 87.8 & .000 & 0.6 & 0.4 & 0.5 & 60.3 & .000 & 0.6 & 0.4 & 0.5 & 70.9 & .000 \\
\hline
\end{tabular}

$p<.05 ; G=$ ganando; $P=$ perdiendo; $E=E m p a t a n d o$

Los jugadores que van ganado obtienen en Wimbledon la media de las variables aces y golpes ganadores superior a la de los otros dos torneos. Por otro lado, en Roland Garros, la media de los errores no forzados es superior en los tenistas que van ganando. Por último, la media de los tenistas que van ganado los partidos obtienen una media superior en las dobles faltas en el Open EEUU.

Finalmente, en la tabla 5 se muestra los resultados de la diferencia entre sets en los indicadores de rendimiento en cada torneo.

Se puede observar que no existen diferencias significativas entre las variables dependientes en los tres torneos analizados con respecto a la diferencia entre sets, excepto en la variable golpes ganadores en Wimbledon y la variable errores no forzados en el Open EEUU.

Los jugadores obtienen en todos los sets en Wimbledon la media de las variables aces y puntos ganados con el servicio superior a la de los otros dos torneos. Por otro lado, en el Open EEUU, la media de dobles faltas es superior en cada uno de los cinco sets.

\section{Discusión}

El objetivo de este trabajo fue comprobar las diferencias existentes entre los indicadores de rendimiento en tres Grand Slams de tenis (Roland Garros, Wimbledon y Open de EEUU), así como el comportamiento de los tenistas ante las diferentes situaciones de juego. A partir de ahí, se ha comprobado que existen indicadores de rendimiento y diferencias significativas en los torneos analizados en cuanto al tipo de torneo, resultado del partido, match status y escasamente en la evolución durante el partido, diferencia entre sets.

Los resultados de este trabajo muestran que hay diferencias significativas en los indicadores de 
Tabla 5. Resultados de la diferencia entre sets con respecto a los indicadores de rendimiento

\begin{tabular}{|c|c|c|c|c|c|c|c|c|c|c|c|c|c|c|c|c|c|c|}
\hline \multirow[t]{3}{*}{$\begin{array}{c}\text { Variables } \\
\text { dependientes }\end{array}$} & \multicolumn{6}{|c|}{ Roland Garros } & \multicolumn{6}{|c|}{ Wimbledon } & \multicolumn{6}{|c|}{ Open EEUU } \\
\hline & $1^{\circ}$ & $2^{\circ}$ & $3^{\circ}$ & $4^{\circ}$ & $5^{\circ}$ & T & $1^{\circ}$ & $2^{0}$ & $3^{\circ}$ & $4^{\circ}$ & $5^{0}$ & T & $1^{\circ}$ & $2^{\circ}$ & $3^{\circ}$ & $4^{\circ}$ & $5^{\circ}$ & T \\
\hline & $\bar{x}$ & $\bar{x}$ & $\bar{x}$ & $\bar{x}$ & $\bar{x}$ & $p$ & $\bar{x}$ & $\bar{x}$ & $\bar{x}$ & $\bar{x}$ & $\bar{x}$ & $p$ & $\bar{x}$ & $\bar{x}$ & $\bar{x}$ & $\bar{x}$ & $\bar{x}$ & $p$ \\
\hline Aces & 1.8 & 1.8 & 1.8 & 1.9 & 1.7 & .654 & 3.0 & 3.3 & 3.2 & 3.1 & 4.7 & .609 & 2.8 & 2.6 & 2.5 & 2.8 & 2.9 & .609 \\
\hline Dobles faltas & 1.0 & 1.1 & 1.1 & 1.1 & 1.0 & .989 & 1.2 & 1.3 & 1.2 & 1.2 & 0.9 & .280 & 1.4 & 1.4 & 1.3 & 1.3 & 1.1 & .760 \\
\hline $\begin{array}{l}\text { \%Primer } \\
\text { servicio }\end{array}$ & 0.6 & 0.6 & 0.6 & 0.6 & 0.6 & .294 & 0.6 & 0.6 & 0.6 & 0.6 & 0.7 & .109 & 0.6 & 0.6 & 0.6 & 0.6 & 0.6 & .673 \\
\hline $\begin{array}{l}\text { P. ganados 1er } \\
\text { servicio }\end{array}$ & 0.7 & 0.7 & 0.7 & 0.7 & 0.7 & .948 & 0.8 & 0.7 & 0.7 & 0.7 & 0.7 & .592 & 0.7 & 0.7 & 0.7 & 0.7 & 0.7 & .997 \\
\hline $\begin{array}{l}\text { P. ganados 2nd } \\
\text { servicio }\end{array}$ & 0.5 & 0.5 & 0.5 & 0.5 & 0.5 & .609 & 0.5 & 0.5 & 0.5 & 0.5 & 0.5 & .914 & 0.5 & 0.5 & 0.5 & 0.5 & 0.5 & .933 \\
\hline $\begin{array}{l}\text { P. de break } \\
\text { salvados }\end{array}$ & 0.4 & 0.5 & 0.5 & 0.4 & 0.4 & .391 & 0.4 & 0.4 & 0.5 & 0.5 & 0.5 & .143 & 0.4 & 0.4 & 0.4 & 0.4 & 0.5 & .622 \\
\hline $\begin{array}{l}\text { P. ganados } 1^{\text {a }} \\
\text { devolución }\end{array}$ & 0.3 & 0.3 & 0.3 & 0.3 & 0.3 & .933 & 0.2 & 0.3 & 0.3 & 0.3 & 0.3 & .534 & 0.3 & 0.3 & 0.3 & 0.3 & 0.3 & .999 \\
\hline $\begin{array}{l}\text { P. ganados } 2^{\text {a }} \\
\text { devolución }\end{array}$ & 0.4 & 0.4 & 0.4 & 0.4 & 0.4 & .510 & 0.4 & 0.4 & 0.4 & 0.4 & 0.4 & .385 & 0.4 & 0.4 & 0.4 & 0.4 & 0.4 & .673 \\
\hline $\begin{array}{l}\text { P. de break } \\
\text { ganadores }\end{array}$ & 0.4 & 0.4 & 0.3 & 0.3 & 0.5 & .211 & 0.3 & 0.3 & 0.3 & 0.3 & 0.3 & .909 & 0.4 & 0.4 & 0.4 & 0.4 & 0.3 & .902 \\
\hline $\begin{array}{l}\text { Golpes ga- } \\
\text { nadores }\end{array}$ & 9.4 & 9.5 & 9.8 & 9.5 & 10 & .895 & 9.4 & 11 & 11 & 11 & 13 & .004 & 10 & 9.9 & 10 & 11 & 11 & .889 \\
\hline $\begin{array}{l}\text { Errores no } \\
\text { forzados }\end{array}$ & 11 & 11 & 10 & 10 & 11 & .175 & 7.3 & 7.7 & 7.9 & 7.5 & 7.9 & .574 & 11 & 10 & 9.9 & 10 & 8.2 & .015 \\
\hline $\begin{array}{l}\text { P. ganados con } \\
\text { el servicio }\end{array}$ & 0.6 & 0.6 & 0.6 & 0.6 & 0.6 & .987 & 0.7 & 0.7 & 0.7 & 0.7 & 0.7 & .972 & 0.6 & 0.6 & 0.6 & 0.6 & 0.6 & .999 \\
\hline $\begin{array}{l}\text { P. ganados con } \\
\text { el resto }\end{array}$ & 0.4 & 0.4 & 0.4 & 0.4 & 0.4 & .987 & 0.3 & 0.3 & 0.3 & 0.3 & 0.3 & .947 & 0.4 & 0.4 & 0.4 & 0.4 & 0.4 & .999 \\
\hline $\begin{array}{l}\text { Total de pun- } \\
\text { tos ganados }\end{array}$ & 0.5 & 0.5 & 0.5 & 0.5 & 0.5 & 1.00 & 0.5 & 0.5 & 0.5 & 0.5 & 0.5 & 1.00 & 0.5 & 0.5 & 0.5 & 0.5 & 0.5 & 1.00 \\
\hline $\begin{array}{l}\text { J. ganados con } \\
\text { el servicio }\end{array}$ & 0.8 & 0.7 & 0.7 & 0.8 & 0.7 & .654 & 0.8 & 0.8 & 0.8 & 0.8 & 0.8 & .816 & 0.8 & 0.8 & 0.8 & 0.8 & 0.8 & .935 \\
\hline $\begin{array}{l}\text { J. ganados con } \\
\text { el resto }\end{array}$ & 0.3 & 0.3 & 0.3 & 0.2 & 0.3 & .645 & 0.2 & 0.2 & 0.2 & 0.2 & 0.2 & .839 & 0.2 & 0.2 & 0.2 & 0.2 & 0.2 & .949 \\
\hline $\begin{array}{l}\text { Total de juegos } \\
\text { ganados }\end{array}$ & 0.5 & 0.5 & 0.5 & 0.5 & 0.5 & 1.00 & 0.5 & 0.5 & 0.5 & 0.5 & 0.5 & 1.00 & 0.5 & 0.5 & 0.5 & 0.5 & 0.5 & 1.00 \\
\hline
\end{tabular}

rendimiento según el tipo de torneo y por lo tanto la superficie de la pista. En el Open UUEE, donde la superficie de la pista es dura, los resultados indican que este torneo se encuentra entre los torneos Roland Garros y Wimbledon. Así, el número de aces, los puntos y juegos ganados con el servicio y los golpes ganadores en pista de hierba o Wimbledon, son superiores a pista dura y a su vez, estos valores son superiores a pistas de tierra o Rolan Garros. Por el contrario, los puntos de break salvados, los puntos ganados con primera y segunda devolución, los errores no forzados y los puntos y juegos ganados al resto en pista de tierra, son superiores a pista dura y a su vez, estos valores son superiores a pistas de hierba. Las conclusiones de diversos estudios van en la línea de estos resultados (Cross \& Pollard, 2009; Barnett \& Pollard, 2007), donde se afirma que la eficacia de conseguir puntos al resto, las oportunidades de rotura del servicio y los errores cometidos son superiores en tierra batida. Además, identificaron que el juego es más lento en tierra batida, el rally es mayor, los partidos tienen una duración mayor (Brown \& O’Donoghue, 2008; Fernández-García, Blanca-Torres, Hernández-García \& Torres-Luque, 2019; Carboch, Siman, Sklenarik \& Blau, 2019) y predomina el estilo de juego defensivo o desde el fondo de la pista (Fernández-García et al., 2012). Por el contrario, otros estudios (FernándezGarcía et al., 2012; Filipcic et al., 2008; Filipcic, Pers \& Klevisar, 2006; Fernández-García et al., 2019) evidenciaron que la velocidad del servicio es superior en las pistas donde la superficie es hierba, que el número de aces es mayor y que las subidas a la red se dan en más ocasiones que en los demás tipos de superficie. Martínez-Gallego 
et al. (2013) pusieron de manifiesto que el juego es más rápido en césped, los puntos tienen un menor número de golpes (Carboch et al., 2019), los partidos tienen una duración menor (Murias, Lanatta, Arcuri \& Laino, 2007; Morante, 2006) y predomina el estilo de juego ofensivo o cerca de la red (Barnett \& Pollard, 2007), tal y como se da a entender en los resultados de la presente investigación. Esta información es valiosa para los tenistas pues deben adaptar sus entrenamientos con el fin de prepararse para soportar partidos de poca duración pero de gran intensidad o largos, disputados y de gran fatiga. Además, los entrenadores deben diseñar tareas específicas de entrenamiento, al igual que estrategias específicas de competición.

Por otro lado, los tenistas que ganan los partidos obtienen una media superior en los tres torneos en todos los indicadores de rendimientos de la investigación frente a los jugadores que pierden, excepto en las dobles faltas, puntos de break salvados y en los errores no forzados. Lo resultados del estudio de Filipcic et al. (2008) afirman que los tenistas, tanto en categoría masculina como femenina, que comenten un número superior de dobles faltas y errores no forzados pierden el partido. Por otro lado, los jugadores que ganan obtienen valores superiores en aces, porcentaje de primer servicio, puntos ganados con primer y segundo servicio, total de golpes y puntos ganadores, al igual que en los resultados de la presente investigación (Filipcic et al., 2008; Katić et al., 2011). Además, los jugadores de tenis que ganan los partidos tienen un mayor índice de puntos de break (O'Donoghue, 2012). Por su parte, Cui et al. (2019) concluyen en su estudio que los jugadores que ganan obtienen porcentajes superiores que los que pierden en puntos ganados con primer y segundo servicio. Dicha información es de gran importancia pues el objetivo principal de todos los jugadores es ganar y estos resultados les aporta información de lo que deben y no deben realizar para hacerlo.

Los jugadores que van ganando los partidos en los tres torneos obtienen una media superior en todos los indicadores de rendimiento, excepto en las dobles faltas, puntos de break salvados y en los errores no forzados. Así, quien va ganando, lleva la iniciativa y tiene más posibilidades de ganar el partido. Ibáñez, Pérez-Goye, CourelIbáñez y García-Rubio (2018) y García-Rubio, Gómez, Lago-Peñas e Ibáñez (2015) concluyen en sus trabajos de futbol profesional que hay una relación significativa entre marcar en primer lugar y ganar el partido. Cui et al. (2019) demostraron que los jugadores con un resultado puntual a favor ganan más puntos de los que pierden. Esta media en los indicadores de rendimiento no es tan favorable cuando el estatus del partido es igualado, siendo claramente inferior cuando se va perdiendo, excepto en las dobles faltas, puntos de break salvados y en los errores no forzados. Ello es entendible, pues obtener una media superior en dobles faltas y errores no forzados conlleva a un déficit en el marcado. Por otro lado, tener una media superior en break salvados significa que a los jugadores les han realizado una gran cantidad de puntos de break, aunque alguno de ellos los haya conseguido salvar. Estos resultados se relacionan con los obtenidos por Cui et al. (2019), los cuales afirman que los jugadores ganan un mayor número de puntos cuando el resultado puntual en su servicio es negativo, mientras que pierden más puntos cuando el resultado puntual durante el resto es positivo. Todo ello conlleva que los jugadores deben adaptar su estilo de juego en función de si van perdiendo, empatando o ganando el partido, así como las estrategias de competición y la toma de decisiones.

Se puede observar en los resultados que no existen diferencias significativas en los tres torneos analizados con respecto a la diferencia entre sets, excepto en la variable golpes ganadores en Wimbledon donde se obtiene un número superior en el quinto set y errores no forzados en el Open EEUU, los cuales se dan en mayor medida en el primer set. Ello puede deberse a que los jugadores inician el partido con una menor exigencia física, técnica, táctica o mental a la que deberían. Sin embargo, a medida avanza el partido la media de los indicadores de rendimiento se estabiliza, no mostrando diferencias entre cada uno de los sets. Así los tenistas deben comenzar el partido con la mayor concentración posible con el objetivo de poder sacar ventaja desde su inicio. No se han encontrado otras investigaciones que estudien la diferencia entre set de indicadores de rendimiento en tenis. Sin embargo, en otro deporte como el baloncesto los resultados revelaron un patrón creciente de coordinación anotadora entre los equipos a medida que los partidos avanzaban, mostrando patrones de coordinación extremadamente altos en el tercer y último cuarto (Prieto-Bermejo, García-Rubio \& Ibánez, 2017). 
Además, Ibáñez, García-Rubio, Rodríguez-Serrano y Feu (2019) analizaron el proceso de juego en baloncesto mostrando un mayor número de ataques en etapas finales de los partidos, con posesiones cortas que terminan en cestas o rebotes, y predominando los ataques posicionales y las defensas individuales de media cancha.

\section{Aplicaciones prácticas}

Los indicadores de rendimiento son característicos de cada tipo de torneo o superficie de juego, condicionando el juego de los tenistas y la preparación específica para cada torneo. El juego en pistas muy rápidas, rápidas y lentas implica la realización de acciones específicas para ganar el partido, estableciéndose estrategias diferentes de competición.

Este estudio ha revelado la importancia del match status y la evolución en el juego en tenis, siendo uno de los primeros estudios en abordarlo. Llevar la iniciativa en el juego es fundamental para conseguir la victoria en tenis, como demuestran las diferencias en los indicadores de rendimiento, por lo que se deben preparar los partidos para iniciar el juego con la máxima intensidad. La forma de juego no varía durante el desarrollo del partido, otorgando un mayor peso a la importancia de llevar la iniciativa en el marcador.

Como prospectiva de futuro, se deberán realizar estudios para comprobar los comportamientos de los deportistas en torneos con características semejantes en cuanto al tipo de pista. Igualmente, será necesario comprobar estas tendencias en la competición femenina, e identificar las diferencias a su vez respecto al juego de los hombres.

\section{Agradecimientos}

Este estudio ha sido parcialmente subvencionado por la Ayuda para Grupos de Investigación (GR18170) del Gobierno Regional de Extremadura (Departamento de Empleo, Empresas e Innovación), con una contribución de la Unión Europea de los Fondos Europeos para el Desarrollo Regional.

\section{Referencias}

Afonso, J., Mesquita, I., \& Palao, J. M. (2005). Relationship between the use of commit-block and the numbers of blockers and block effectiveness. International Journal of Performance Analysis in Sport, 5(2), 36-45. https://doi.org/10.1080/24748668.2005.11868326

Akpinar, S., Devrilmez, E., \& Kirazci, S. (2012). Coincidenceanticipation timing requirements are different in racket sports. Perceptual and Motor Skills, 175(2), 581-593. https://doi.org/10.2466/30.25.27.PMS. 115.5.581-593

Anguera, M. T., \& Hernández-Mendo, A. (2016). Avances en estudios observacionales de Ciencias del Deporte desde los mixed methods. Cuadernos de Psicología del Deporte, 16(1), 17-30. https://revistas.um.es/cpd/article/view/254261

Ato, M., López, J. J., \& Benavente, A. (2013). Un sistema de clasificación de los diseños de investigación en psicología. Anales de Psicología, 29(3), 1038-1059. https://doi.org/10.6018/analesps.29.3.178511

Barnett, T., \& Pollard, G., (2007). How the tennis court surface affects player performance and injuries. Medicine and Science in Tennis, 12(1), 34-37.

Bloomfield, J. R., Polman, R. C. J., \& O'donoghue, P. G. (2005). Effects of score-line on team strategies in FA Premier League Soccer. Journal of Sports Sciences, 23(2), 192-193.

Brown, E., \& O`Donoghue, P. (2008). Efecto del género y la superficie en la estrategia del tenis de élite. Coaching and Sport Science Review, 15(46), 11-13.

Carboch, J., Siman, J., Sklenarik, M., \& Blau, M. (2019). Match Characteristics and Rally Pace of Male Tennis Matches in Three Grand Slam Tournaments. Physical Activity Review, (7), 49-56.

Courel, J., Mc Robert, A., Ortega, E., \& Cárdenas, D. (2014). The impact of match status on game rhythm in NBA basketball. In Comunicación presentada en el Congress of the European College of Sport Science.

Cowden, R. G., Fuller, D. K., \& Anshel, M. H. (2014). Psychological predictors of mental toughness in elite tennis: an exploratory study in learned resourcefulness and competitive trait anxiety. Perceptual and motor skills, 119(3), 661-678. https://doi.org/10.2466/30.PMS.119c27z0

Cross, R., \& Pollard, G. (2009). Grand Slam men's singles tennis 19912009. Serve speeds and other related data. Coaching \& Sport Science Review, 16(49), 8-10. http://www.physics.usyd.edu.au.

Cubo, S. (2011). La investigación experimental. En S. Cubo; B. Marín \& J.L. Ramos (Eds). Métodos de investigación y análisis de datos en ciencias sociales y de la salud (pp.235-326). Madrid: Ediciones Pirámide.

Cui, Y., Liu, H., Liu, H., \& Gómez, M. Á. (2019). Data-driven analysis of pointby-point performance for male tennis player in Grand Slams. Motricidade, 15(1), 49-61. http://dx.doi.org/10.6063/motricidade.16370

Fernández, J., Méndez, A., Pluim B. M., Fernández-García, B., \& Terrados, N. (2006). Aspectos físicos y psicológicos del tenis en competición. Archivos de Medicina del Deporte, 13(116), $451-454$.

Fernández-Fernández, J., Kinner, V., \& Ferrauti, A. (2010). The physiological demands of hitting and running in tennis on different surfaces. The Journal of Strength \& Conditioning Research, 24 (12), 3255-3264. https://doi.org/10.1519/JSC.0b013e3181e8745f

Fernández-García, Á. I., Blanca-Torres, J. C., Hernández-García, R., \& Torres-Luque, G. (2019). Análisis de las variables estadísticas relacionadas con el servicio en tenis masculino de alto rendimiento en categoría junior y absoluto. Cultura, Ciencia y Deporte, 14(42), 289-295. http://dx.doi.org/10.12800/ccd.v14i42.1342

Fernández-García, A. I., Torres-Luque, G., Sánchez-Pay, A., \& Fradua, L. (2012). Influencia del tipo de superficie en las estadísticas de competición del tenis de alto rendimiento. In Proceedings of VII Congreso Internacional de la Asociación Española de Ciencias del Deporte (pp. 412-413)

Ferrauti, A., Bergeron, M. F., Pluim, B. M., \& Weber, K. (2001). 
Physiological responses in tennis and running with similar oxygen uptake. European Journal of Applied Physiology, 85, 27-33. https://doi.org/10.1007/s004210100425

Filipcic, A., Pers, J., \& Klevisar, A. (2006). Comparison between Young male and female tennis players in terms of time and movement characteristics. In: Proceedings of 4th World Congress of Science and Racket Sports, 2006, Madrid, Spain (pp. 87-90)

Filipcic, T., Filipcic, A., \& Berendijas, T (2008). Comparison of game characteristics of male and female tennis players at Roland Garros 2005. Acta Universitatis Palackianae Olomucensis. Gymica, 38(3), 21-28.

Furlong, J.D. (1995). The service in lawn tennis: how important is it?. Science and racket sports. In: T. Reilly, M. Hughes; Lees, A. (Eds) Science and Racket Sports I, (pp 266-271). London, England: E \& FN Spon.

García-Rubio, J., Gómez, M. Á., Lago-Peñas, C., \& Ibáñez, J. S. (2015). Effect of match venue, scoring first and quality of opposition on match outcome in the UEFA Champions Leaque. International Journal of Performance Analysis in Sport, 15(2), 527-539. https://doi.org/10.1080/24748668.2015.11868811

Gillet, E., Leroy, D., Thouvarecq, R., \& Stein, J. F. (2009). A notational analysis of elite tennis serve and serve-return strategies on slow surface. The Journal of Strength \& Conditioning Research, 23 (2), 532-539. https://doi.org/10.1519/JSC.0b013e31818efe29

Gómez, M. Á., Alarcón-López, F., \& Ortega-Toro, E. (2015). Analysis of shooting effectiveness in elite basketball according to match status. Revista de Psicología del Deporte, 24(3), 0037-0041. https://ddd.uab.cat/record/145126

Hughes, M., \& Franks, I. (2007). The essentials of performance analysis: an introduction. Routledge.

Ibáñez, S. J., García-Rubio, J., Rodríguez-Serrano, D., \& Feu, S. (2019). Development of a Knockout Competition in Basketball: A Study of the Spanish Copa del Rey. Frontiers in psychology, 10. https://doi.org/10.3389/fpsyg.2019.02457

Ibáñez, S. J., Pérez-Goye, J. A., Courel-Ibáñez, J., \& GarcíaRubio, J. (2018). The impact of scoring first on match outcome in women's professional football. International Journal of Performance Analysis in Sport, 18(2), 318-326. https://doi.org/10.1080/24748668.2018.1475197

Jones, P. D., James, N., \& Mellalieu, S. D. (2004). Possession as a performance indicator in soccer. International Journal of Performance Analysis in Sport, 4(1), 98-102. https://doi.org/10.1080/24748668.2004.11868295

Katić, R., Milat, S., Zagorac, N., \& Đurović, N. (2011). Impact of game elements on tennis match outcome in Wimbledon and Roland Garros 2009. Collegium antropologicum, 35(2), 341-346.

Kovacs, M. (2007). Tennis physiology. Training the competitive athlete. Sport Medicine, 37(3), 189-198.

Lago,C.,(2009). Theinfluence ofmatchlocation, quality ofopposition, and match status on possession strategies in professional association football. Journal of sports sciences, 27(13), 14631469. https://doi.org/10.1080/02640410903131681

Lago, C. \& Martín, R. (2007). Determinants of possession of the ball in soccer. Journal of sports sciences, 25(9), 969-974. https://doi.org/10.1080/02640410600944626
Lees, A. (2003). Science and the major racket sports: a review. Journal of Sports Sciences, 21(9), 707-732. https://doi.org/10.1080/0264041031000140275

Martínez-Gallego, R., Gumán, J.F., James, N., Pers, J., Ramon-Llin, J., \& Vuckovic, G. (2013). Movement Characteristics of Elite Tennis Player son Hard Courts with Respet to the Direction of Ground Strokes. Journal of Sports Science and Medicine, 10(1), 275281. https://www.ncbi.nlm.nih.gov/pmc/articles/PMC3761832/

Monteiro, R., Mesquita, I., \& Marcelino, R. (2009). Relationship between the set outcome and the dig and attack efficacy in elite male Volleyball game. International Journal of Performance Analysis in Sport, 9(3), 294-305. https://doi.org/10.1080/24748668.2009.11868486

Montero, I., \& León, O.G. (2007). A guide for naming research studies in Psychology. International Journal of Clinical and Health Psychology, 7(3), 847-862. http://www.redalyc.org/articulo.oa?id=33770318

Morante, S. (2006). Training Recommendations based on Match Characteristics of Professional Singles Tennis. Medicine and Science in Tennis. 17(3): 10-13.

Murias, J.M., Lanatta, D., Arcuri, C.R., \& Laino, F.A. (2007). Metabolic and functional responses playing tennis on different surfaces. Journal Strength and Condition Research. 21(1): 112-117.

O'Donoghue, P. (2012). Break points in Grand Slam men's singles tennis. International Journal of Performance Analysis in Sport, 12(1), 156-165. https://doi.org/10.1080/24748668.2012.11868591

O'Donoghue, P., \& Ingram, B. (2001). A notational analysis of elite tennis strategy. Journal of Sports Sciences, 19(2), 107-115. https://doi.org/10.1080/026404101300036299

Piles, J., \& Crespo, M. (2012). Tactics for Elite level Mens TennisPart 1. ITF Coaching and Sport Science Review, 56(20), 9-10.

Prieto-Bermejo, J., García-Rubio, J., \& Ibáñez, S. J. (2017). Scoring coordination patterns in basketball international championships of national teams. Revista de psicología del deporte, 26(3), 0027-32. https://ddd.uab.cat/record/171677

Reid, M., MMurtrie, D., \& Crespo, M (2010). The relationship between match statistics and top 100 ranking in profesional mens's tennis. International Journal of Performance Analysis in Sport, 10(2), 131-138. https://doi.org/10.1080/24748668.2010.11868509

Sánchez-Pay, A., Torres-Luque, G., Fernandéz-Garcia, Á. I., SanzRivas, D., \& Andrés, J. M. P. (2017). Differences in game statistics between winning and losing for male wheelchair tennis players in Paralympics Games. Motriz: Revista de Educação Física, 23(3). https://doi.org/10.1590/s1980-6574201700030011

Smekal, G., von Duvillard, S. P., Rihacek, C., Pokan, R., Hofmann, P., Baron, R., \& Bachl, N. (2001). A physiological profile of tennis match play. Medicine and Science in Sports and Exercise, 33(6), 999-1005. https://journals.Iww.com/acsm-msse/Fulltext/2001/06000/A

Unierzyski, P., \& Wieczorek, A. (2004). Comparison of tactical solutions and game patterns in the finals of two grand slam tournaments in tennis. In: A. Lees, J.F. Kahn, I.W. Maynard (Eds). Science and Racket Sports III, (pp 169-174). London and New York: Routledge; Taylor \& Francis Group. 